\title{
LA APROXIMACIÓN DE LAS ESTRATEGIAS DE LA UE Y DE LOS EEUU EN LA LUCHA CONTRA LA PROLIFERACIÓN DE ARMAS DE DESTRUCCIÓN MASIVA
}

\author{
Milagros Álvarez ${ }^{1}$ \\ Universidad de Barcelona (UB)
}

\begin{abstract}
Resumen:
El cambio en el seno de la Administración estadounidense tras las elecciones de 2008 y la consiguiente adopción de nuevos documentos estratégicos en la lucha contra la proliferación de armas de destrucción masiva suscitan nuevamente el interés de su comparación con los planteamientos defendidos por la UE, al objeto de identificar la posible emergencia de nuevos márgenes de coincidencia entre ambos actores internacionales y, con ello, el impulso a nuevos compromisos en el régimen de no proliferación. La comparación revela tres tendencias fundamentales: estancamiento en el proceso de mejora y perfeccionamiento de la regulación prevista para las armas químicas y biológicas, desarrollo selectivo del régimen de no proliferación nuclear, y consolidación de la cooperación informal y ad hoc como instrumento contra la proliferación de ADM.
\end{abstract}

Palabras clave: No proliferación, armas de destrucción masiva, Estados Unidos, UE.

Title in English: "An Approach to the EU and the USA Strategies in the Fight against Proliferation of Weapons of Massive Destruction”.

\begin{abstract}
:
The change within the U.S. administration after the elections of 2008 and the subsequent adoption of new strategic documents in the fight against proliferation of weapons of mass destruction raise, again, the interest of comparison with the approaches advocated by the EU; this can make possible to identify the likely emergence of new agreement between them and thus, their impetus to new commitments in the non-proliferation regime. The comparison reveals three basic trends: stagnation in the process of improving and refining the rules laid down for chemical and biological weapons, selective development of nuclear non-proliferation regime, and consolidation of informal and ad hoc cooperation as a tool against the proliferation of WMD.
\end{abstract}

Keywords: nonproliferation, weapons of mass destruction, EU, United States.

Copyright $@$ C UNISCI, 2012.

Las opiniones expresadas en estos artículos son propias de sus autores, y no reflejan necesariamente la opinión de UNISCI. The views expressed in these articles are those of the authors, and do not necessarily reflect the views of UNISCI.

\footnotetext{
1 Milagros Álvarez Verdugo es Profesora Titular de Derecho Internacional Público de la Universitat de Barcelona (UB).

E-mail: malvarez@ub.edu.

http://dx.doi.org/10.5209/rev_UNIS.2012.n30.40700
} 


\section{Introducción}

La adopción en 2003 de la Estrategia de la UE contra la proliferación de armas de destrucción masiva (ADM) marcó un punto de inflexión en el papel a desarrollar por esta organización en un ámbito de interés y relevancia internacional evidente. No exenta de limitaciones y ambigüedades, el documento formalizó las líneas maestras de la acción de la UE e identificó los instrumentos y mecanismos de utilización europea preferente. El contexto político internacional en el momento de su adopción también fue relevante: concretamente, 2003 fue quizás el año más convulso desde la perspectiva de las relaciones trasatlánticas, tras la invasión de Iraq liderada por los EEUU y la fractura política que esa acción originó entre los Estados de la UE. ${ }^{2}$ En ese contexto, la comparación entre el documento entonces adoptado por la UE y la Estrategia de los EEUU en la materia, publicada en 2002, ponía de manifiesto importantes diferencias en las políticas de estos dos actores internacionales, con consecuencias para el desarrollo del propio régimen de no proliferación de ADM.

A punto de cumplirse diez años desde la adopción de la Estrategia europea, el tiempo transcurrido permite advertir cuáles han sido los ejes de acción prioritarios de la UE en aplicación de la misma y la modulación que esa aplicación pudiera estar produciendo en el propio 'marco teórico' diseñado en 2003. Además, el cambio en el seno de la Administración estadounidense tras las elecciones de 2008 ha originado la adopción de nuevos documentos estratégicos que suscitan nuevamente el interés de su comparación con los planteamientos de la UE, al objeto de identificar la posible emergencia de nuevos márgenes de coincidencia entre ambos y, con ello, el impulso a nuevos compromisos en el régimen de no proliferación de ADM, incluidos sus mecanismos de aplicación.

Se trata de una comparación que excluye las diferencias motivadas por la distinta naturaleza de estos dos actores internacionales y, en buena medida, obvia los problemas institucionales de la UE para definir y desarrollar una política de no proliferación. ${ }^{3}$ De hecho, la UE no es parte de ninguno de los tratados vigentes que regulan las ADM o aspectos relacionados con el objetivo de no proliferación y cuenta con una limitada participación en los foros internacionales que abordan otras cuestiones conexas. Así mismo, cuando hablamos de la UE estamos haciendo referencia a un sujeto de derecho internacional que, en términos políticos, engloba a un conjunto de Estados con posiciones y estatus distintos en el ámbito que nos ocupa y que se visualizan, en mayor o menor medida, con ocasión de las conferencias internacionales en las que participan. Todo ello incide en el protagonismo de la UE y en su capacidad propositiva en el ámbito de la lucha contra la proliferación de ADM. Sin embargo, esas mismas características convierten a la UE en un ejemplo relevante de la tipología de consensos que pudieran estar forjándose respecto al posible desarrollo del régimen de no proliferación de las ADM y, en ese proceso, advertir en que medida los planteamientos defendidos desde el gobierno estadounidense están teniendo incidencia.

Con ese propósito, organizaremos esta contribución en tres apartados. El primero repasará sucintamente las diferencias que separaban la Estrategia de la UE con la aprobada en 2002 por los EEUU, y las consecuencias que ello generaba desde la perspectiva del régimen internacional vigente en materia de ADM. El segundo apartado identificará las características fundamentales de los nuevos documentos de estrategia adoptados por el gobierno

\footnotetext{
${ }^{2}$ Fractura que erosionaba la cohesión en el seno de la UE y dañaba sus aspiraciones de convertirse en un actor global relevante. Ver Van Ham, P., "The European Union's WMD Strategy and the CFSP: a critical analysis", EU Non-Proliferation Consortium, Non-Proliferation Papers, no. 2 (Sept. 2011), p. 3.

${ }^{3}$ Sobre esta cuestión y las nuevas posibilidades que ofrece la reforma del Tratado de Lisboa, ver Zwolski, K., "The External Dimension of the EU's Non-proliferation Policy: Overcoming Inter-institutional Competition", European Foreign Affairs Review, vol. 16, no. 3 (2011), pp. 325-340.
} 
estadounidense en esta materia, destacando sus coincidencias y diferencias con las posiciones defendidas por la anterior administración republicana. Sobre esta base, el tercer apartado se centrará en delimitar cuáles son los actuales márgenes de potencial coincidencia entre la UE y los EEUU y, con ello, el tipo de desarrollo del régimen de no proliferación que puede generar el acuerdo entre estos dos actores internacionales.

\section{La comparación de la estrategia europea con la estrategia estadounidense de 2002}

La comparación entre la Estrategia de la UE y la adoptada en 2002 por los EEUU revelaba profundas diferencias respecto al diagnóstico, los objetivos y el tratamiento de los riesgos y retos asociados a las $\mathrm{ADM}^{4}$ Esas diferencias han estado en el origen de algunas de las dificultades experimentadas durante la última década por el régimen de no proliferación pero también explican algunas de sus actuales tendencias de desarrollo.

Como punto de partida, la primera y principal diferencia era el enfoque adoptado por cada uno de estos actores, que podía calificarse de 'reactivo' (EEUU) y 'proactivo' (UE). Así, el planteamiento de los EEUU pertenecía a los parámetros clásicos de la defensa del Estado: identificación de enemigos (Estados hostiles y grupos terroristas) y protección contra estos, muy especialmente contra sus capacidades en $\mathrm{ADM}^{5}$ Consecuentemente, los EEUU adoptaban un enfoque de legítima defensa que explicaba la preeminencia asignada a las medidas de contraproliferación y a las medidas de carácter unilateral. ${ }^{6}$ Significativamente, la estrategia estadounidense no otorgaba ningún papel al desarme en el combate contra las $\mathrm{ADM}$, erosionando con ello el pacto principal que fundamenta el régimen de no proliferación. En el caso de la Estrategia europea, tanto su diagnóstico de las amenazas asociadas a las ADM como los objetivos que persigue la UE en este campo, están condicionados por la propia naturaleza de la Unión: una organización internacional que aborda el tratamiento de problemas a través de mecanismos multilaterales y que, además, está compuesta por una pluralidad diversa de Estados miembros. Ello explica que la Estrategia de la UE no se plantee como objetivo la desaparición de este tipo de armas y que ni siquiera califique de amenaza la propia existencia de ADM sino el riesgo de proliferación de las mismas, agravado en la actualidad por el fenómeno del terrorismo. ${ }^{7}$ En ese contexto, los objetivos enunciados por la

\footnotetext{
${ }^{4}$ The National Strategy to Combat WMD, December 2002, at www.fas.org/irp/offdocs/nspd/nspd-wmd.pdf. Para un análisis detallado de la comparación entre ésta y la Estrategia de la UE nos remitimos a nuestra publicación "Comparing U.S. and E.U. Strategies Against Weapons of Mass Destruction: Some Legal Consequences", Annual Survey of International \& Comparative Law, vol. 11 (2005), p. 119-140.

${ }^{5}$ En palabras de Cirincione, "Bush Administration has changed the issue from "what" to "who". Ver, Cirincione, J.: "Symposium on Security \& Liberty: Speech: Proliferation Threats and Solutions", Notre Dame Journal of Law, Ethics \& Public Policy, vol. 19 (2005), p. 344. Este diagnóstico de amenazas implicaba, de hecho, un importante cambio por comparación a estrategias anteriores de los EEUU. Por ejemplo, la Estrategia de Seguridad Nacional de 1999 afirmaba lo siguiente: "WMD is a global concern that transcends national borders. It is the greatest potential threat to global stability and security. Proliferation threatens to provide rogue states, terrorist and international crime organizations with the means to inflict terrible damage on the US, our allies, and US citizens and troops abroad." Ver, The White House, A National Security Strategy for a New Century, (December 1999), p. 2.

6 Según explicaba un destacado representante de la Administración republicana, el Gobierno Bush “is reinventing the nonproliferation regime it inherited' y su política revelaba "that a robust use of the sovereign authorities that we [US], and our allies, have at our disposal can bring about real results". Ver, Bolton, John R., "The Bush Administration's Forward Strategy for Nonproliferation", Chicago Journal of International Law, vol. 5, no. 2 (Winter 2005), p. 395.

${ }^{7}$ Ver, Estrategia de la UE contra la proliferación de armas de destrucción masiva (en adelante, Estrategia UEADM), parágrafo 1.
} 
UE en su documento de estrategia son incluso limitados: evitar, disuadir, detener y, cuando sea posible, eliminar los programas de proliferación que suscitan preocupación a escala mundial. ${ }^{8}$ Estos objetivos permiten además a la UE un cierto grado de apreciación subjetiva, pues no incluye referencia alguna a la institución u órgano competente para calificar de ese modo un determinado programa de proliferación. La posición de la UE, aunque universal, evita así limitar sus posibilidades de actuación a los casos calificados por el Consejo de Seguridad como 'amenaza a la paz y a la seguridad internacionales', y deja abierta la posibilidad de su participación en otras acciones de carácter multilateral.

Las diferencias entre la Estrategia de la UE y el documento homólogo adoptado en 2002 por los EEUU generaban un límite a la posible elaboración de nuevas obligaciones jurídicas e incluso a la aplicación de las normas internacionales vigentes en materia de ADM. De hecho, en la medida que para los EEUU la amenaza no procedía ni de la existencia de estas armas ni tan siquiera del riesgo de proliferación, sino de las características de sus poseedores, se hacía imposible la generación de normas internacionales de alcance general. Únicamente en la conexión entre ADM y terrorismo podía identificarse algún punto de encuentro entre ambas Estrategias y ese ha sido, en efecto, el ámbito temático donde se han producido novedades desde la perspectiva del régimen internacional de las ADM: la Resolución 1540 adoptada por el Consejo de Seguridad en 2004, a instancias entre otros de los EEUU y de la UE, obliga a todos los Estados del globo a adoptar y aplicar determinadas medidas legislativas y controles nacionales con el objetivo de evitar el acceso de los actores no estatales y grupos terroristas a las ADM y a sus sistemas vectores. ${ }^{9}$ Ahora bien, la diferente confianza en las instituciones multilaterales que se observaba al comparar la Estrategia de la UE con la adoptada en 2002 por los EEUU tenía también su correlativo reflejo en la Resolución del Consejo de Seguridad: aunque en ella se reconoce que los Estados pueden necesitar asistencia para la correcta aplicación de las obligaciones que establece, la articulación de esa asistencia no se organiza en términos multilaterales sino que se hace depender de la cooperación voluntaria que puedan ofrecer los Estados. ${ }^{10}$

La estrecha y subjetiva delimitación del ámbito de aplicación de la Estrategia estadounidense de 2002 (Estados hostiles) también tenía repercusiones en la determinación del cumplimiento de las obligaciones que impone a los Estados el régimen de no proliferación, muy especialmente las establecidas en tratados carentes de mecanismos institucionalizados de verificación y control. En la medida que los EEUU sólo manifestaban interés en los casos de proliferación protagonizados por Estados hostiles, enviaba con ello un claro mensaje político a la comunidad internacional: el incumplimiento de las obligaciones en el ámbito de las ADM podía compensarse con una relación política 'saludable' con los EEUU. De hecho, el desinterés estadounidense por los supuestos de proliferación imputables

\footnotetext{
${ }^{8}$ Ver, Estrategia UE-ADM, parágrafo 2.

${ }^{9}$ Cuestión esta que ya hemos abordado en otro momento y a cuya investigación nos remitimos. Ver, Álvarez Verdugo, M. (2007), Incidencia del Consejo de Seguridad sobre el régimen jurídico de las armas nucleares, Bosch Ed., Barcelona.

${ }^{10}$ Vid. S/RES/1540 (2004), parágrafo 7. Ejemplos de esta cooperación voluntaria son el programa de los EEUU para la reducción del riesgo nuclear (Programa Nunn-Lugar) o la iniciativa adoptada por el G-8 en junio de 2002 , en ambos casos dirigidos a minimizar los riesgos de proliferación y mejorar las condiciones de seguridad de los materiales nucleares en los territorios de la antigua Unión Soviética. También la UE ha arbitrado fórmulas e iniciativas de cooperación voluntaria para facilitar la aplicación de la Resolución 1540. Ver. Acción Común 2006/419/PESC de 12 de junio de 2006 y Acción Común 2008/368/PESC de 14 de mayo de 2008, de apoyo a la aplicación de la Resolución del Consejo de Seguridad de las Naciones Unidas 1540 (2004) en el marco de la ejecución de la Estrategia de la UE contra la proliferación de armas de destrucción masiva (DO L 165 de 17.6.2006 y DO L 127 de 15.5.2008).
} 
a Estados no hostiles generaba efectos para la UE, que difícilmente podría llegar a considerar tales programas como susceptibles de suscitar preocupación a nivel mundial.

También desde la perspectiva de la aplicación de las obligaciones jurídicas vigentes, concretamente los mecanismos internacionales de verificación y control, las Estrategias de la UE y de los EEUU mostraban profundas diferencias. Frente a la posición de la UE favorable a la efectiva utilización, mejora y desarrollo de este tipo de mecanismos, las ambiguas referencias que se contienen a este respecto en la Estrategia estadounidense de 2002 se materializaron en una actuación claramente contraria al desarrollo de mecanismos internacionales e institucionalizados de verificación y control. Baste recordar la oposición de EEUU, en diciembre de 2001, a la adopción de un Protocolo a la Convención sobre armas bacteriológicas y toxínicas (CABT), ${ }^{11}$ su decisión de reformular el mandato de negociación del Tratado para la prohibición de la producción de materiales fisibles ${ }^{12}$ o sus argumentos para rechazar la ratificación del Tratado de prohibición completa de ensayos nucleares. ${ }^{13} \mathrm{~A}$ cambio, optaba por medidas unilaterales o, como mucho, por fórmulas multilaterales de carácter selectivo y ad hoc, de las que la Iniciativa de Seguridad contra la Proliferación lanzada en mayo de 2003 por el Presidente Bush es su mejor ejemplo. ${ }^{14}$

Finalmente, los aspectos más controvertidos de la Estrategia estadounidense se situaban en el ámbito de la aplicación coactiva. El texto identificaba el uso unilateral de la fuerza armada, incluidas las armas nucleares de los EEUU, como una medida de contraproliferación, contraviniendo con ello la regulación jurídica internacional vigente del uso de la fuerza y del derecho de legítima defensa $\mathrm{y}$, adicionalmente, abrogando una garantía de seguridad anteriormente sostenida por los EEUU: el compromiso de no utilizar su fuerza nuclear contra Estados no poseedores de armas nucleares. ${ }^{15}$ Sin asemejarse a este tipo de planteamientos, no es menos cierto que la Estrategia de la UE trata de manera ambigua la cuestión de la adopción de medidas coercitivas frente a situaciones de proliferación y tampoco es clara sobre el papel que a tal efecto debe desempeñar el Consejo de Seguridad. Así, el documento señala la

\footnotetext{
${ }^{11}$ Protocolo que regulaba un mecanismo internacional de verificación y control para este Tratado. Ver Dando, Malcolm R. (2002): Preventing Biological Warfare: the failure of American leadership, Great Britain, Palgrave; Tucker, Jonathan B.: "The BWC New Process: A Preliminary Assessment," Nonproliferation Review, vol. 11, no. 1 (2004), p. 26-39 y Ward, Kenneth D.: “The BWC Protocol: Mandate for Failure," Nonproliferation Review, vol. 11, no. 2 (2004), p. 183-199.

12 Eliminando el objetivo de conseguir un 'tratado efectivamente verificable', dado que a juicio de la administración republicana eso no era posible sin comprometer intereses nacionales de seguridad. Vid. U.S. Department of State and U.S. Agency for International Development. Fiscal Year 2006 Joint Performance Plan (February 2005), p. 121, at www.state.gov/documents/organization/41623.pdf.

${ }^{13}$ Entre otros, su valoración de que los mecanismos de verificación fijados por este Tratado podían comprometer intereses nacionales de seguridad de los EEUU. Ver, Hewitson, P.: "Between Empire and Community: The United States and Multilateralism 2001-2003: A Mid-Term Assessment: Arms Control: Nonproliferation and Reduction of Nuclear Weapons: Risks of Weakening the Multilateral Nuclear Nonproliferation Norm," Berkeley Journal of International Law, vol. 21, no. 3 (2003), p. 451.

${ }^{14}$ Dos perspectivas distintas sobre las virtualidades y efectos de esta Iniciativa son la de Samuel E. Logan: "The Proliferation Security Initiative: Navigating the Legal Challenges", Journal of Transnational Law \& Policy, vol. 14, no. 2 (Spring 2005), p. 253 y la de Daniel H. Joyner: "The Proliferation Security Initiative: Nonproliferation, Counterproliferation, and International Law", The Yale Journal of International Law, vol. 30, no. 2 (Summer 2005), p. 507. La UE no respaldó esta Iniciativa hasta junio de 2004, tras la adopción de la Resolución 1540 del Consejo de Seguridad. Ver, Council of the EU, Non-Proliferation Support of the Proliferation Security Initiative, Brussels, 1 June 2004, 10052/04 (Presse 189).

${ }^{15}$ Compromisos recogidos, entre otros, en el documento "Principles and Objectives adopted by the NPT 1995 Review Conference" y en el Documento NPT/CONF.2000/6, sobre "Security Assurances". A este respecto, ver Ware, Alyn, "Nuclear Proliferation: Rule of Force or Rule of Law? Legal Responses to Nuclear Threats from Terrorism, Proliferation, and War", Seattle Journal for Social Justice, vol. 2, no. 1 (Fall 2003 / Winter 2004), p. 251.
} 
posibilidad de sanciones de diverso tipo, incluidas medidas que implican el uso de la fuerza, y sin embargo se limita a afirmar que "el Consejo de Seguridad debe desempeñar un papel protagonista". ${ }^{16}$ La ambigüedad elegida por la UE sólo puede interpretarse como una vía que deja a esta la posibilidad de adoptar medidas coercitivas, también las que implican el uso de la fuerza armada, sin la explícita autorización del Consejo de Seguridad, abriendo un espacio de potencial coincidencia con la Estrategia estadounidense que contradice el régimen jurídico internacional vigente y no favorece la aplicación de sus normas, tampoco de las que establecen obligaciones en el ámbito de la no proliferación de ADM. ${ }^{17}$

\section{Principales novedades y características de la estrategia definida por la Administración Obama en relación a las ADM}

La administración demócrata de los EEUU, a través de la 'National Strategy for Countering Biological Threats' adoptada en 2009, la 'Nuclear Posture Review' (NPR) de abril de 2010 y la 'National Security Strategy' de mayo del mismo año, ha identificado las nuevas líneas maestras de la política estadounidense en materia de ADM. ${ }^{18}$ El mayor cambio operado en esos documentos es, quizás, el lenguaje y el tono empleados en su redacción, lo que ha generado importantes expectativas de avance en el reforzamiento del régimen internacional vigente y en el respaldo de los EEUU a una aplicación objetiva de sus normas. El análisis detallado de esos documentos pone ciertamente de manifiesto cambios importantes respecto a la estrategia de la anterior administración republicana y, con ello, la renovación de compromisos anteriormente asumidos por los EEUU con la comunidad internacional. La constatación de esos cambios no impide sin embargo poder afirmar una sustantiva coincidencia por cuanto respecta a sus líneas de acción prioritarias.

En efecto, los documentos actualmente vigentes encuadran la definición de las amenazas asociadas a las ADM de manera distinta a como lo hacía la Estrategia adoptada en 2002 y sugieren una aproximación global a los riesgos que estas armas representan. En palabras de la nueva Estrategia de Seguridad Nacional, "there is no greater threat to the American people than weapons of mass destruction, particularly the danger posed by the pursuit of nuclear weapons by violent extremists and their proliferation to additional states". ${ }^{19}$ En esa línea, la NPR de 2010 califica como amenazas más apremiantes el terrorismo nuclear y la proliferación nuclear, si bien individualiza como preocupación específica el riesgo de proliferación nuclear en países enfrentados con los Estados Unidos, sus socios y aliados, y la comunidad internacional más amplia. ${ }^{20}$. Un enfoque incluso más omnicomprensivo parece adoptar la Estrategia de 2009 contra las amenazas biológicas, calificando de tales a las armas biológicas, en su conjunto, y también su uso o proliferación por Estados y actores no estatales. ${ }^{21}$ El desarrollo posterior de este documento pone no obstante de manifiesto la centralidad en la prevención de ataques biológicos de carácter terrorista y en la manera de responder ante enfermedades a gran escala causadas por agentes de este tipo, con independencia de que las mismas tengan un origen natural o intencionado. De este modo, la Estrategia de 2009 contra las amenazas biológicas traduce la posición adoptada por la

\footnotetext{
${ }^{16}$ Estrategia de la UE-ADM, parágrafo 15.

${ }^{17}$ Sin entrar en otros desarrollos o referencias posibles, creemos que en este punto es suficiente con recordar el art. 24 de la Carta de las Naciones Unidas y las competencias exclusivas que el Capítulo VII de la misma asigna al Consejo de Seguridad.

${ }^{18}$ Documentos todos ellos publicados en el sitio www.whitehouse.gov.

${ }^{19}$ National Security Strategy, p. 4.

${ }^{20}$ NPR, op. cit., p. 3.

${ }^{21}$ National Strategy for Countering Biological Threats, p. 2.
} 
administración demócrata estadounidense, que ha desistido de la posibilidad de firmar un protocolo anexo a la CABT por considerar imposible la verificación internacional efectiva del cumplimiento de dicha Convención.

El lenguaje empleado en estos tres textos revela un cambio de enfoque en relación con las ADM: son las ADM en su conjunto las que merecen el calificativo de amenaza así como el riesgo de proliferación de las mismas. Pero a partir de aquí las prioridades de la acción estadounidense permanecen casi inalteradas, y sigue centrada en la prevención y lucha contra el terrorismo nuclear y la proliferación nuclear, muy especialmente la protagonizada por Estados enfrentados - ya no hostiles - a los Estados Unidos, sus socios y la comunidad internacional más amplia. La flexibilidad con que puede interpretarse esta última expresión hace de ella una novedad de importancia relativa desde la perspectiva de su impacto efectivo en las decisiones de los EEUU.

La prioridad de la proliferación y del terrorismo nuclear en la política de los EEUU se revela incluso con mayor claridad en el único apartado de la nueva Estrategia de Seguridad Nacional dedicado a las ADM. Bajo el título "Detener la propagación de armas nucleares y biológicas y asegurar los materiales nucleares", el apartado omite mención alguna a las armas químicas o a los riesgos asociados a estas, ${ }^{22}$ y el único párrafo relativo al combate contra las amenazas biológicas lo hace desde la perspectiva de la prevención y protección de la salud de las personas, las capacidades para hacer frente a las enfermedades originadas por este tipo de agentes y la represión de los posibles responsables, sin referencia alguna a las armas biológicas o al tratado internacional que las regula. ${ }^{23} \mathrm{Y}$ es que ocho de los nueve párrafos de este apartado abordan aspectos vinculados con las armas nucleares y la proliferación nuclear. ${ }^{24}$

La centralidad 'nuclear' de la política estadounidense en materia de ADM tiene en la NPR de 2010 su desarrollo más concreto. ${ }^{25}$ Este documento dota de contenido al objetivo formulado por el Presidente Obama en su discurso de Praga de abril de 2009, conseguir un mundo libre de armas nucleares. ${ }^{26}$ Se trata sin duda alguna de su novedad más significativa,

\footnotetext{
${ }^{22}$ La actualidad de los riesgos asociados a las armas químicas tiene su ejemplo más significativo en el incumplimiento por EEUU, Rusia y Libia de la fecha fijada para la destrucción completa de estas armas, 29 de abril de 2012, en virtud de la Convención sobre la prohibición del desarrollo, la producción, el almacenamiento y el empleo de armas químicas y sobre su destrucción. A este respecto, ver Kelle, A.: "Chemical weapons destruction deadline missed", Bulletin of the Atomic Scientists, 24 April 2012, en http://www.thebulletin.org/web-edition/columnists/alexander-kelle/chemical-weapons-destruction-deadlinemissed.

${ }^{23}$ Ver National Security Strategy, p. 23-24.

${ }^{24}$ En ellos se enuncian cuatro objetivos de alcance general (procurar alcanzar un mundo sin armas nucleares, reforzar el TNP, asegurar los materiales y armas nucleares vulnerables y apoyar la energía nuclear para usos pacíficos) y también objetivos específicos respecto a los casos de Corea del Norte e Irán: lograr la desnuclearización de la península de Corea y prevenir el desarrollo del arma nuclear por parte de Irán.

${ }^{25}$ La NPR enuncia y desarrolla los siguientes objetivos: evitar la proliferación nuclear y el terrorismo nuclear, reducir el papel de las armas nucleares de los EEUU en la estrategia de seguridad nuclear, mantener la disuasión y la estabilidad estratégica con niveles de fuerzas nucleares reducidas, fortalecer la disuasión regional y la confianza de los aliados y socios estadounidenses, y mantener un arsenal nuclear estable, seguro y efectivo. Ver, NPR, p. 2. La Nonproliferation Review dedica el No. 1 de su volumen 18 (de marzo de 2011), al análisis de la nueva NPR. Específicamente, la Introducción elaborada por Scott D. Sagan y J. Vayman (p. 17-37) aborda las diferencias y similitudes entre este documento y la NPR adoptada en 2001 (no publicada) por el Gobierno Bush y las diferentes vías por las que la nueva NPR puede influenciar el comportamiento de otros actores internacionales.

${ }^{26}$ En dicho discurso, el Presidente Obama dibujó una ambiciosa agenda en materia de desarme nuclear, no proliferación y contraterrorismo que ha sido objeto de distintas interpretaciones pero cuya aplicación en la práctica necesita de múltiples y diversas realizaciones concretas, de los EEUU y de la comunidad internacional
} 
pues reincorpora a la agenda política internacional la consecución de un compromiso jurídico vigente que durante años resultó impensable. ${ }^{27}$ Ahora bien, la desaparición de estas armas se hace depender en la NPR de un conjunto de condiciones que trasladan tal posibilidad a un futuro cuando menos lejano. ${ }^{28}$ Mientras tanto, la contribución estadounidense a la consecución de ese objetivo final se sitúa lejos de las obligaciones que impone el art. VI del TNP pues se limita a la reducción condicionada del arsenal nuclear y a la reformulación del papel asignado a este tipo de armamentos. ${ }^{29}$

Así, y desde una perspectiva cuantitativa, la posible reducción del arsenal nuclear de los EEUU seguirá estando sujeta según la NPR a parámetros clásicos de disuasión y estabilidad estratégica que además, se afirma, deben preservarse a un triple nivel, regional, internacional y bilateral. ${ }^{30} \mathrm{Sin}$ renunciar por tanto al mantenimiento de un arsenal nuclear seguro, protegido y eficaz, ${ }^{31}$ la estrategia estadounidense sí muestra una nueva predisposición hacia tratados internacionales que inciden en el desarrollo y control de este tipo de armas: celebración de tratados bilaterales con Rusia para la reducción de armas estratégicas, voluntad de ratificar el Tratado de prohibición completa de ensayos nucleares, y compromiso de iniciar negociaciones para la firma de un Tratado relativo a la prohibición de la producción de material fisible. Hasta la fecha, el único de estos compromisos que se ha materializado ha sido la firma y entrada en vigor del nuevo Tratado START entre Rusia y los EEUU.

Respecto al papel asignado a las armas nucleares, la NPR reformula la política de empleo de estas armas por parte de los EEUU, actualizando las garantías de seguridad negativa declaradas en 1995 por los cinco Estados poseedores de armas nucleares partes del TNP y eliminando, a priori, la respuesta nuclear ante un ataque con armas químicas o biológicas. Además, y aunque la NPR reserva a EEUU el derecho a replantearse estas garantías de no respuesta nuclear si las circunstancias lo aconsejaran en caso de ataque biológico, ${ }^{32}$ la utilización de armas nucleares únicamente se contempla "en circunstancias extremas para defender los intereses vitales de los Estados Unidos o sus aliados", lenguaje que recuerda lo afirmado por el TIJ en su Opinión Consultiva de 1996 sobre la legalidad de la amenaza o empleo de las armas nucleares y convierte en instrumento de último recurso a este tipo de armas.

en su conjunto. Algunas de ellas las analiza Perkovich, G., "The Obama Nuclear Agenda One Year After Prague", Carnegie Endowment for International Peace, Policy Outlook (31 Mar. 2010).

${ }^{27}$ Ver, Gutierrez Espada, C. \& Cervell Hortal, M.J., "La nueva estrategia nuclear estadounidense: ¿un futuro libre de armas nucleares?", REDI, vol. LXII (2010-2011), p. 292.

${ }^{28}$ Esas condiciones son: interrupción exitosa de la proliferación de armas nucleares, mayor transparencia en los programas y los medios de los principales países participantes, métodos de verificación y tecnologías capaces de detectar las violaciones a las obligaciones del desarme, medidas de cumplimiento suficientemente fuertes y creíbles para disuadir las violaciones, y la resolución de las diferencias regionales que pueden motivar a estados rivales a adquirir y mantener armas nucleares. NPR, p. 48-49.

${ }_{29}$ Especialmente crítico con los contenidos de la NPR desde la perspectiva de las obligaciones de desarme nuclear establecidas en el TNP es Moxley Jr., Ch.J.: “Obama's Nuclear Posture Review: An Ambitious Program For Nuclear Arms Control But A Retreat From The Objective Of Nuclear Disarmament", Fordham International Law Journal, vol. 34, no. 4 (2011), p. 734-775.

${ }^{30}$ La NPR insiste en varias ocasiones en la necesidad de mantener con niveles reducidos de fuerza nuclear la disuasión estratégica y la estabilidad con Rusia y China, así como en la exigencia de fortalecer los vínculos de seguridad bilateral y regional que mantienen los EEUU en Europa, Asia y Oriente Próximo. NPR, p. 4-6 y 19-35. Sobre la tensión entre la política de disuasión nuclear definida en la NPR y el Derecho internacional, ver Nagan, W.P. \& Slemmens, E.K.: "Developing U.S. Nuclear Weapons Policy and International Law: The Approach of the Obama Administration”, Tulane Journal of International \& Comparative Law, vol. 19 (2010), p. 41-77.

${ }^{31}$ En la NPR se renuncia a la fabricación de nuevas armas nucleares pero no a la modernización de las ya existentes, distinción que en la práctica puede dar lugar a muy distintas interpretaciones. Ver, Moxley Jr., Ch.J., “Obama's Nuclear Posture ...", op. cit., p. 761-762.

${ }^{32}$ NPR, op. cit., p. 16. 
La reconducción, en los términos que hemos señalado, de la cuestión del desarme nuclear y del papel asignado a su arsenal nuclear coloca a los EEUU, según la NPR, "en una posición mucho más fuerte" para persuadir al conjunto de Estados parte del TNP de la necesidad de adoptar medidas adicionales con las que reforzar el régimen de no proliferación y mejorar la seguridad de los materiales nucleares a nivel mundial. ${ }^{33}$ El planteamiento no deja de resultar hasta cierto punto paradójico, pues la declaración política de estar dispuesto a abordar, en un futuro incierto, el cumplimiento de una obligación jurídica vigente (celebrar negociaciones de buena fe sobre medidas eficaces relativas al desarme nuclear - art. VI TNP )$^{34}$ junto con la abrogación de políticas de empleo de las armas nucleares que contravenían, desde distintas perspectivas, el régimen jurídico internacional, 'son' las razones que según EEUU fortalecen su posición y le otorgan una auctoritas renovada que le legitima para pedir el acuerdo del resto de Estados a la adopción de medidas que sí implican nuevas restricciones, compromisos y obligaciones jurídicas. ${ }^{35}$

En efecto, un primer grupo de medidas adicionales que juzgan necesarias tiene por objeto el reforzamiento del régimen de no proliferación e incide en cuatro ámbitos distintos de la regulación que se contiene en el TNP. ${ }^{36}$

Primero, el mecanismo de verificación y control sobre los Estados no poseedores de armas nucleares, previsto en su artículo III, que se entiende debe completarse mediante la ratificación por dichos Estados de un Protocolo Adicional a sus acuerdos de salvaguardia con el OIEA. Adicionalmente, la NPR se muestra favorable al incremento de los recursos financieros e instrumentos a disposición de este Organismo para llevar a cabo sus tareas de verificación. ${ }^{37}$

Segundo, el derecho de denuncia del TNP regulado en su artículo VIII, impidiendo su ejercicio a cualquier Estado parte en situación de incumplimiento con las obligaciones que para él se deriven de este Tratado.

Tercero, el control de las exportaciones nucleares, reforzando los regímenes nacionales y multilaterales existentes así como las restricciones a las transferencias de tecnologías susceptibles de doble uso, especialmente las relacionadas con actividades de enriquecimiento de uranio y reprocesamiento de combustible nuclear. A tal efecto, y junto al incremento de los fondos estadounidenses para la financiación de programas de cooperación bilateral, la NPR manifiesta el apoyo de los EEUU a la creación de un fondo conjunto para la aplicación de la

\footnotetext{
${ }^{33}$ NPR, op. cit., p. 7, primer párrafo.

${ }^{34}$ Además de paradójico, el planteamiento estadounidense es contradictorio con la literalidad del artículo VI del TNP pues implica una interpretación parcial y selectiva de la obligación jurídica que se establece en dicha disposición: "Cada Parte en el Tratado se compromete a celebrar negociaciones de buena fe sobre medidas eficaces relativas a la cesación de la carrera de armamentos nucleares en fecha cercana y al desarme nuclear, y sobre un tratado de desarme general y completo bajo estricto y eficaz control internacional".

${ }^{35}$ Sobre el impacto efectivo de la nueva política de no proliferación nuclear estadounidense, vid. Müller, H.: “A Nuclear Nonproliferation Test - Obama's Nuclear Policy and the 2010 NPT Review Conference", Nonproliferation Review, vol. 18 no. 1 (March 2011), p. 219-236. A juicio del autor, el impacto fundamental fue generar un ambiente en el que el compromiso 'era lo correcto', lo que, en consecuencia, hizo posible la adopción de un documento final en la última Conferencia de revisión del TNP. Ahora bien, los contenidos de ese documento final son el mínimo común denominador entre las posiciones defendidas por los Estados poseedores de armas nucleares y el Movimiento de países no alineados, con la única excepción de la sección relativa a Oriente Próximo, hecho que refleja la mutua neutralización entre las posiciones defendidas por unos y otros y la ausencia de cualquier progreso sustancial.

${ }^{36}$ NPR, op. cit., p. 9-10.

37 El documento recoge el compromiso de los EEUU de incrementar sus aportaciones ordinarias y extraordinarias al presupuesto del OIEA, sin precisar no obstante más detalles al respecto (NPR p. 9-10).
} 
Resolución 1540 del Consejo de Seguridad y propone convertir en organización internacional la Iniciativa contra la Proliferación Nuclear.

Cuarto, el alcance del derecho al uso pacífico de la energía nuclear enunciado en el art. IV del TNP, mediante la apuesta por mecanismos multilaterales de diversa naturaleza que hagan innecesario el desarrollo individualizado por los Estados de las fases más sensibles del ciclo nuclear, muy especialmente las relacionadas con el enriquecimiento de uranio.

El segundo grupo de medidas adicionales que plantea la NPR hace referencia a la seguridad de los materiales nucleares, con el objetivo primordial de prevenir el terrorismo nuclear. Sin mención alguna a los tratados internacionales vigentes en la materia, ${ }^{38}$ la cuestión se aborda bajo la premisa de que garantizar la seguridad de estos materiales es una responsabilidad estrictamente estatal y, en ese contexto, la NPR recoge el compromiso de reforzar e incrementar los programas estadounidenses para la cooperación bilateral y asistencia a terceros Estados (Nunn-Lugar, Global Threat Reduction Initiative, International Nuclear Material Protection and Cooperation Program, etc.). El documento sólo recoge una iniciativa de cooperación multilateral ad hoc, la celebración de cumbres mundiales sobre seguridad nuclear; iniciativa que hasta la fecha se ha concretado en la celebración de dos cumbres, en 2010 y $2012 .^{39}$

\section{Nuevos ámbitos de coincidencia UE/EEUU para el desarrollo y aplicación del Régimen de no Proliferación de ADM}

La UE ha consolidado durante los últimos años una notable acción en aplicación de la Estrategia adoptada en 2003 contra la proliferación de ADM manteniendo, en líneas generales, una coherencia esencial con el diagnóstico y los instrumentos entonces definidos. Dicha acción, que se recoge en los Informes semestrales preparados por el Consejo, se ha acompañado también de un esfuerzo de actualización y revisión de las prioridades de la UE en este ámbito. ${ }^{40}$ Además, y con el propósito de reforzar el papel de la UE en la lucha contra la proliferación de ADM, el Consejo aprobó en diciembre de 2008 el documento titulado "Nuevas líneas de acción de la UE en el combate contra la proliferación de ADM y sus

\footnotetext{
38 Que existen y que, en ciertos casos, tienen un número de Estados parte que convendría incrementar. Ver Convención sobre protección física de los materiales nucleares, de 3 de marzo de 1980 (BOE núm. 256 de 25/10/1991) con 145 Estados parte (la enmienda a esta Convención, hecha en 1995, sólo cuenta con 55 Estados parte, incluida España); Convención sobre Seguridad Nuclear, de 20 de septiembre de 1994 (BOE núm. 236 de 30/9/1996) con 74 Estados parte; Convención conjunta sobre seguridad en la gestión del combustible gastado y sobre seguridad en la gestión de desechos radiactivos, de 5 de septiembre de 1997 (BOE núm. 97 de 23/04/2001) con 42 Estados parte.

${ }^{39}$ Ver, Nuclear Security Summit Final Communique y Nuclear Security Summit Final Work Plan, Washington DC, 12-13 Abril 2010, en http://fpc.state.gov/c35775.htm; y Seoul Communiqué - 2012 Seoul Nuclear Security Summit, (26-27 Marzo 2012), en www.un.org/disarmament/content/spotlight. Existen valoraciones bien distintas sobre el impacto efectivo que estas cumbres pueden llegar a tener en las regulaciones y compromisos nacionales sobre seguridad nuclear y también sobre la conveniencia de que en ellas se adopten directrices y estándares de aplicación mundial, posibilidad esta última que por el momento rechaza el gobierno estadounidense. Ver, Guarino, D.P.: "U.S. Official Rejects Call For International Nuclear Security Standards", Global Security Newswire, (1 Mayo 2012), en www.nti.org/gsn/article/us-official-rejects-call-internationalnuclear-security-standards/.

${ }_{40}$ Mientras que la presentación de los informes semestrales se realiza con regularidad desde 2004, la actualización del listado de prioridades se ha hecho en cuatro ocasiones: en diciembre de 2004, 2005 y 2006 y en junio de 2008. Para la consulta de esos informes semestrales y de las listas actualizadas de las prioridades de la UE, ver www.consilium.europa.eu/eeas/foreign-policy/non-proliferation,-disarmament-and-export-control/documentation/documents?lang=en\#Bookmark4.
} 
sistemas vectores" el cual, sin cuestionar la vigencia de la Estrategia de 2003, enuncia nuevos principios y objetivos específicos. ${ }^{41}$

La comparación entre la Estrategia de la UE contra la proliferación de ADM, a la luz de la acción desplegada para su aplicación, con los nuevos documentos de estrategia estadounidense en esta materia revela nuevas similitudes que podrían favorecer la actuación conjunta de estos dos actores internacionales en algunos ámbitos concretos. Esas nuevas similitudes no sólo tienen su razón de ser en los actuales planteamientos del gobierno de los EEUU sino, también, en la evolución experimentada por la propia UE.

Una primera aproximación se produce en la definición de las amenazas asociadas a las ADM, pues la UE parecería estar incorporando la sutil diferenciación entre enfoque general y prioridades de actuación que observábamos en los actuales textos estadounidenses. Así, las Nuevas líneas de acción adoptadas en 2008 califica a las ADM en manos de 'states of concern or terrorists/non state actors' como la mayor de las amenazas a la seguridad que pueden enfrentar los europeos. ${ }^{42}$ La afirmación, que incorpora una priorización que no constaba en documentos anteriores de la UE, no modifica per se los contenidos de la Estrategia de 2003 y, de hecho, está precedida por la consideración de que la proliferación de ADM y de sus sistemas vectores, en general, es un riesgo para la seguridad de los europeos mucho mayor de lo que lo era en 2003. Tomado en su conjunto, el planteamiento del documento de 2008 ofrece no obstante dos matizaciones relevantes. Una, el texto parecería reflejar una mayor y más profunda toma de conciencia por parte de la UE de la importancia y los retos que plantea la proliferación de estos armamentos. ${ }^{43}$ Dos, la propia experiencia reciente de la UE, involucrada directamente en casos concretos de amenaza de proliferación nuclear (léase, las negociaciones con Irán), trasladan a sus documentos lo que podríamos denominar un cierto pragmatismo que le permite individualizar y, por tanto, priorizar sus actuaciones dentro de la problemática general de la proliferación de ADM y del enfoque omnicomprensivo con que su Estrategia de 2003 aborda esta cuestión.

La acción desarrollada por la UE desde 2003 también revela un cierto cambio en el tratamiento de las amenazas asociadas a las armas biológicas, acercándola a los planteamientos de los EEUU. ${ }^{44}$ Ciertamente, la UE sigue desplegando iniciativas dirigidas a favorecer la universalización y plena aplicación de la Convención sobre armas bacteriológicas y toxínicas (CABT). ${ }^{45}$ Sin embargo, parecería haber renunciado a la posibilidad de desarrollar un mecanismo de verificación y control de este tratado, aceptando el planteamiento estadounidense de que no es posible un régimen de verificación efectivo en relación a las armas biológicas, y ha trasladado sus iniciativas al ámbito del establecimiento y consolidación

\footnotetext{
41 Ver Council of the European Union: "New lines for action by the European Union in combating the proliferation of weapons of mass destruction and their means of delivery", de 17 de diciembre de 2008 (Documento 17172/08). En adelante, utilizaremos la abreviatura 'UE-New lines' para referiremos a este documento.

${ }^{42}$ UE-New lines, p. 3.

${ }^{43}$ O, como mínimo, ese pretendía ser el objetivo principal de este documento. Ver Van Ham, P., "The European Union's WMD Strategy...", op.cit. p. 5.

${ }^{44}$ Cambio detectado por algunos autores ya en 2006. Ver, Zanders, J.P., "The European Union and the 6th Review Conference", The European Union and the 2006 BTWC Review Conference, Chaillot Paper No. 93 (November 2006), p. 93-118.

45 Acción Común 2006/184/PESC de 27 de febrero de 2006 y Acción Común 2008/858/PESC de 10 de noviembre de 2008, en apoyo de la Convención sobre armas bacteriológicas y toxínicas en el marco de la ejecución de la Estrategia de la UE contra la proliferación de armas de destrucción masiva (DO L 65 de 7.3.2006 y DO L 302 de 13.11.2008).
} 
de mecanismos eficaces de fomento de la confianza respecto al cumplimiento de la CABT. ${ }^{46}$ Paralelamente, los aspectos relacionados con la salud y la seguridad biológicas están adquiriendo un creciente protagonismo en el ámbito de preocupaciones preferentes de la UE, abriendo con ello un espacio de potencial coincidencia y acción conjunta con los EEUU. ${ }^{47}$

El control de las exportaciones de bienes y tecnologías de doble uso y la lucha contra su tráfico ilícito ocupa, así mismo, una parte cada vez más importante de la acción de la UE. ${ }^{48}$ No en vano tres de las siete líneas de acción recogidas en el documento adoptado por la UE en 2008 tienen una relación directa con estas cuestiones: intensificar la cooperación y asistencia a terceros Estados para mejorar sus sistemas nacionales de control y sus políticas de no proliferación, combatir las transferencias intangibles de conocimiento y know-how e intensificar los esfuerzos dirigidos a impedir los flujos de proliferación. ${ }^{49}$ A tal efecto, la UE ha desarrollado y modificado sus normas internas ${ }^{50}$ y ha impulsado diversas acciones en el plano internacional. Entre esas acciones destacan los programas de asistencia a terceros Estados para la aplicación de las obligaciones fijadas en este ámbito por la Resolución 1540 del Consejo de Seguridad, los programas de apoyo, vía el OIEA, a la mejora de las capacidades de los Estados en la lucha contra el tráfico ilícito, ${ }^{51}$ y la creación en distintas regiones del globo de los denominados 'Centros de Excelencia' contra el tráfico ilícito de materiales químicos, biológicos, radiológicos y nucleares. ${ }^{52}$

En cualquier caso, la mayor aproximación entre las posiciones de la UE y de los EEUU se produce en aspectos concretos del régimen de no proliferación y seguridad nuclear. Así, y como se reflejaba en la posición común para la Conferencia de revisión de 2010 del TNP, la UE sostiene desde hace tiempo y coincide con los EEUU en la necesidad de generalizar la ratificación de un Protocolo adicional como estándar de verificación conforme al art. III del

\footnotetext{
${ }^{46}$ Algunas de estas iniciativas se explican en el Informe semestral sobre la aplicación de la Estrategia UE-ADM (2010-II), Consejo de la UE - Documento 17080/10. Ver también, Plan de Acción de la UE relativo a las armas biológicas y toxínicas, complementario de la Acción conjunta de la UE de apoyo a la CABT (DO C 57 de 9.3.2006) y Decisión 2011/429/PESC del Consejo, de 18 de julio de 2011, relativa a la posición de la Unión Europea para la Séptima Conferencia de Revisión de los Estados Partes en la Convención sobre la prohibición del desarrollo, la producción y el almacenamiento de armas bacteriológicas (biológicas) y toxínicas y sobre su destrucción (CABT) (DO L 188 de 19.7.2011, p. 42).

47 Acción Común 2008/307/PESC del Consejo, de 14 de abril de 2008, de apoyo a las actividades de la Organización Mundial de la Salud en el ámbito de la bioseguridad y la bioprotección de los laboratorios, en el marco de la Estrategia de la Unión Europea contra la proliferación de armas de destrucción masiva (DO L 106, de 16.04.2008, p. 17). Dos de los tres documentos de trabajo presentados por la UE durante los trabajos previos a esta conferencia abordaban cuestiones de este tipo: "'EU capacities to respond to CBRN attacks and CBRN incidents" y "EU cooperative initiatives to improve bio-safety and bio-security". Vid. Consejo de la UE, Informe semestral sobre la aplicación de la Estrategia UE-ADM (2010-II), Documento 17080/10, p. 32.

${ }^{48}$ La Estrategia de la UE ya recogía de hecho su compromiso con el fortalecimiento de las políticas y prácticas de control de las exportaciones dentro y fuera de sus fronteras. Vid. Estrategia UE-ADM, párrafos 14 y 19.

${ }^{49}$ UE-New lines, p. 5, 11 y 14.

50 Reglamento (CE) no 428/2009 del Consejo, de 5 de mayo de 2009, por el que se establece un régimen comunitario de control de las exportaciones, la transferencia, el corretaje y el tránsito de productos de doble uso (DO L 134, de 29 de mayo de 2009). Respecto a los antecedentes de este Reglamento y sus déficits desde la perspectiva del establecimiento de un auténtico régimen europeo común para el control de estas exportaciones, ver Micara, A.G., "Current features of the European Union Regime for Export Control of Dual-Use Goods", JCMS, vol. 50, no. 4 (2012), p. 578-593.

${ }^{51}$ La última es la Decisión 2010/585/PESC del Consejo, de 27 de septiembre de 2010, sobre el apoyo a las actividades del OIEA en los ámbitos de la seguridad y la verificación nucleares y en el marco de la aplicación de la Estrategia de la UE contra la proliferación de armas de destrucción masiva (DO L 259 de 1.10.2010).

${ }^{52}$ Iniciativa esta última en línea con el Plan de Acción adoptado por la Comisión el 24 de junio de 2009 y que alcanza, actualmente, a ocho regiones del globo. Ver, Consejo de la UE, Informe semestral sobre la aplicación de la Estrategia UE-ADM 2009-II, p. 5 (Doc. 17387/09), 2010-II, p. 39-40 (Doc. 17080/10) y 2011-II, apdo. 5 (Doc. 7062/12).
} 
TNP y en la de complementar y fortalecer el régimen de denuncia previsto en dicho Tratado. También por cuanto respecta al uso pacífico de la energía nuclear, la posición europea es favorable a fórmulas multilaterales del ciclo del combustible nuclear e insiste en la necesidad de desarrollar un régimen de control de las exportaciones nucleares que garantice la no desviación de estos bienes y tecnologías hacia actividades de proliferación. ${ }^{53}$

El acercamiento entre la UE y los EEUU en la defensa de iniciativas concretas contra la proliferación de ADM no excluye la persistencia de dos diferencias fundamentales entre ambos. Más allá de los nuevos énfasis que se aprecian en la acción de la UE, ésta sigue manteniendo una visión omnicomprensiva de la problemática que rodea a las ADM y por tanto una acción, quizás de intensidad desigual, pero en todo caso simultánea en apoyo a los tres tratados internacionales que vertebran su régimen jurídico internacional y la lucha contra la proliferación de cada una de estas armas. ${ }^{54}$ Así mismo, dicha acción se desarrolla mayoritariamente en el marco de las organizaciones e instituciones internacionales competentes, circunstancia que probablemente merma la visibilidad internacional de la UE pero que es coherente con su defensa del multilateralismo como manera de hacer frente a los retos que plantean las ADM. Por parte estadounidense, la estrategia vigente incrementa sin duda el respaldo de este país a la acción multilateral institucionalizada, pero sigue mostrando una sólida confianza en la eficacia de su acción bilateral directa y en su propia capacidad de liderazgo para el tratamiento internacional de problemas conectados con la proliferación de ADM a través de mecanismos de cooperación informal y ad hoc. Junto a esta, la otra diferencia significativa con la UE es la marginalización de las armas químicas y biológicas en la estrategia estadounidense y su comprensión de las amenazas de la proliferación en términos esencialmente nucleares, impulsando con ello un desarrollo parcial y selectivo del régimen jurídico internacional de las ADM.

\section{Consideraciones finales}

El desarrollo del régimen internacional vigente en materia de ADM depende de múltiples factores que exceden de la simple identificación de las posibilidades de acuerdo, en cada momento, entre los EEUU y la UE. Ahora bien, la importancia de las relaciones trasatlánticas desde la perspectiva de la acción exterior de la UE y la relevancia de estos dos actores en la escena internacional hacen de sus márgenes de coincidencia un buen indicador de hacia dónde puede dirigirse la regulación internacional de este tipo de armas y la cooperación internacional contra la proliferación. En este sentido, la comparación realizada permite afirmar la presencia de tres tendencias fundamentales.

\footnotetext{
${ }^{53}$ Decisión 2010/212/PESC del Consejo de 29 de marzo de 2010 relativa a la posición de la Unión Europea en la Conferencia de las Partes del año 2010 encargada del examen del Tratado sobre la no proliferación de las armas nucleares (DO L 90 de 14.04.2010). Para una valoración de los resultados obtenidos por la UE en esta Conferencia de revisión, ver Sturm, P.: “The EU's performance at the 2010 NPT Review Conference”, ISIS Europe, European Security Review, no. 50 (July 2010) y Dee, M.: "Standing together or Doing the Splits? Evaluating European Union Performance in the Nuclear Non-proliferation Treaty Review Negotiations", European Foreign Affairs Review, vol. 17, no. 2 (2012), pp. 189-211.

${ }^{54}$ Además de las ya citadas en relación al TNP y al CABT, vid. Decisión 2012/166/PESC del Consejo, de 23 de marzo de 2012, de apoyo a las actividades de la Organización para la Prohibición de las Armas Químicas (OPAQ) en el marco de la aplicación de la Estrategia de la UE contra la proliferación de armas de destrucción masiva (DO L 87 de 24.3.2012) y Posición Común 2007/469/PESC del Consejo, de 28 de junio de 2007, relativa a la Conferencia de Revisión de 2008 de la Convención sobre la Prohibición del Desarrollo, la Producción, el Almacenamiento y el Empleo de Armas Químicas y sobre su Destrucción (DO L 176 de 6.7.2007).
} 
Primera, y desde la perspectiva del desarrollo conjunto del régimen jurídico internacional de las ADM, se observa un cierto estancamiento en el proceso de mejora y perfeccionamiento de la regulación prevista para las armas químicas y biológicas. En el caso de las primeras las consecuencias de este estancamiento pueden valorarse de menos graves, dadas las características de su Convención internacional de referencia y de la Organización internacional encargada del control de su aplicación, la OPAQ. No ocurre lo mismo respecto a las armas biológicas, cuya prohibición sigue necesitada de un mecanismo internacional de verificación que garantice la destrucción de estos arsenales y ofrezca al conjunto de Estados parte de la CABT la adecuada confianza sobre el correcto y generalizado cumplimiento de las obligaciones establecidas en este tratado.

Segunda tendencia, y en sentido contrario, esos mismos márgenes de coincidencia entre la UE y los EEUU sí apuntan hacia un desarrollo del régimen de no proliferación nuclear centrado, esto sí, en el fortalecimiento de los mecanismos de control sobre los Estados no poseedores de armas nucleares y en una cierta reinterpretación del alcance del derecho a la energía nuclear para usos pacíficos que afecta, básicamente, a esos mismos Estados. Sin medidas específicas complementarias en el ámbito de las obligaciones sobre desarme, este tipo de desarrollo del régimen de no proliferación nuclear significaría una reformulación del contenido asignado por el TNP a dos de sus tres pilares básicos y, por tanto, la redefinición del pacto entre Estados poseedores y no poseedores de armas nucleares consagrado en dicho tratado.

Finalmente, y desde la perspectiva de las formas de cooperación, la tercera tendencia a la que apunta la comparación de los documentos de estrategia estadounidense con la Estrategia y la acción desplegada por la UE es en el sentido de la consolidación de la cooperación informal y ad hoc como instrumento contra la proliferación de ADM. Si bien es cierto que estos mecanismos de cooperación han jugado tradicionalmente un papel fundamental en el control de las exportaciones de bienes y tecnologías de doble uso, ahora se extienden también al tratamiento de otros aspectos tales como el tráfico ilícito o la seguridad nuclear. Sin infravalorar sus efectos positivos en la generación de consensos y en el impulso a la generalización de prácticas estatales de no proliferación, estos nuevos foros e instrumentos informales de cooperación no incorporan elementos de conexión con las organizaciones internacionales competentes en la materia ni con el acervo convencional existente, circunstancia que, además de solapamientos, puede operar en detrimento de la universalización y aplicación efectiva de los tratados internacionales vigentes y del papel asignado a tal efecto a la cooperación internacional institucionalizada. 